\title{
Integrating Monitoring of Volume Status and Blood Volume-Controlled Ultrafiltration into Extracorporeal Kidney Replacement Therapy
}

\author{
Sebastian Zschätzsch' \\ Manuela Stauss-Grabo \\ Adelheid Gauly $\mathbb{D}^{2}$ \\ Jennifer Braun ${ }^{2}$ \\ 'Center for Kidney and Blood Pressure \\ Diseases, Georg-Haas-Dialysis Center, \\ Giessen, Germany; ${ }^{2}$ Fresenius Medical \\ Care, Global Medical Office, Bad \\ Homburg, Germany
}

\begin{abstract}
Purpose: Volume management in hemodialysis (HD) requires the ability to assess volume status objectively and determine treatment strategies that achieve euvolemia without compromising hemodynamic stability. The aim of this study was to compare dialysis with and without blood volume-controlled ultrafiltration (UF) in combination with body composition monitoring, and to evaluate indicators for adequate dialysis $(\mathrm{Kt} / \mathrm{V})$, ultrafiltration volume, fluid status, and the occurrence of intradialytic morbid events (IME).

Patients and Methods: Patients undergoing hemodialysis or on-line hemodiafiltration with support of a blood volume monitor (BVM) - a feedback control device integrated into the 5008 and 6008 HD systems - were enrolled. Patients received treatment for four weeks using the 6008 CAREsystem and the BVM $(6008+)$. Data on dialysis dose $(\mathrm{Kt} / \mathrm{V})$, UF volume and predialysis fluid status were documented. This data was also documented retrospectively for four weeks with $(5008+)$ and without $\left(5008^{-}\right)$the use of the BVM with the 5008 system. Comparisons were analyzed using linear mixed models.

Results: Twenty-four patients were enrolled. Kt/V was unaffected by blood volumecontrolled UF (5008- vs 5008+: $p=0.230)$ and was equally achieved with both HD systems (5008+ vs $6008+: p=0.922)$. The UF volume and fluid status achieved were comparable, independent of the use of UF control with BVM (5008- vs 5008+; UF volume: $p=0.166$; fluid overload: $p=0.390$ ) or the HD system (5008+ vs $6008+$ : UF volume: $p=0.003$; fluid overload: $p=0.838$ ), except for UF volume being higher in the $6008+$ phase. IMEs occurred in less than $3 \%$ of treatments, with no difference between study phases.

Conclusion: This study demonstrates that a clinical approach to kidney replacement therapy that tracks volume status and manages intradialytic fluid removal by blood volumecontrolled UF delivers adequate dialysis without compromising fluid removal. It maintains volume status and ensures low incidence of IMEs.
\end{abstract}

Keywords: fluid status, blood volume monitoring, body composition monitoring, dialysis dose, intradialytic morbid events

\section{Introduction}

In patients with kidney failure receiving kidney replacement therapy (KRT), volume management is a major challenge. Decreasing kidney function and gradual loss of diuresis go along with an increased risk of volume overload. This can contribute to hypertension and heart failure and is associated with increased mortality risk. ${ }^{1-3}$ Therefore, avoidance and reduction of volume excess mainly accumulating in the extracellular space are recommended to improve blood pressure control ${ }^{4}$ and reduce
Correspondence: Adelheid Gauly Fresenius Medical Care Deutschland $\mathrm{GmbH}$, Global Medical Office, ElseKröner-Strasse 3, Bad Homburg, 61352, Germany

Tel +4961726092260

Email adelheid.gauly@fmc-ag.com 
mortality risk. ${ }^{2}$ The use of aggressive ultrafiltration strategies in hemodialysis (HD) is usually limited because cardiovascular comorbidities may lead to intolerance of marked blood volume reduction during dialysis and may enhance intradialytic hemodynamic instability. ${ }^{5}$ Volume depletion may cause intradialytic hypotension and other intradialytic morbid events, which are also associated with adverse patient outcomes. ${ }^{6,7}$

It is therefore crucial to determine optimal volume status, employ assessment methods and implement treatment strategies to bring patients carefully to euvolemia without, for example, excess ultrafiltration rates, which have been identified as a mortality risk factor. ${ }^{8,9}$

Management of the patient's volume status includes its control over the long term and during single dialysis sessions. Long-term management may involve the use of clinical and technical approaches, including, among others, bioimpedance spectroscopy, ${ }^{10,11}$ lung ultrasound, ${ }^{12}$ natriuretic peptides $^{13}$ and clinical assessment. ${ }^{14}$ Controlling intradialytic volume status is challenging due to restricted HD session time and the requirement of achieving a tolerable ultrafiltration rate to remove accumulated fluid.

Blood volume changes during hemodialysis depend on the balance between ultrafiltration and plasma refilling. These usually do not occur at the same rate, leading mostly to decreased blood volume.

Intradialytic morbid events, including hypotensive episodes, may occur in response to blood volume reduction when cardiovascular compensatory mechanisms are insufficient. The concept of critical blood volume, whether in absolute ${ }^{15}$ or relative terms, ${ }^{16}$ specifies a value below which the risk of intradialytic hypotension or other intradialytic morbid events is elevated. This suggests blood volume monitoring during HD sessions and counteraction by adjusting the ultrafiltration rate to avoid reaching the critical value.

Advanced hemodialysis machines make it possible to noninvasively monitor blood volume relative to the start of dialysis (relative blood volume, RBV), and linked feedback control algorithms can adjust the ultrafiltration rate to prevent RBV from falling below a patient's individual critical RBV ( $\left.\mathrm{RBV}_{\text {crit }}\right)$.

Today, the use of blood volume monitoring and UF control is not yet established as a standard in hemodialysis. With the clinical study presented here, a therapeutic approach to assessing volume status with bioimpedance spectroscopy and the use of blood volume-controlled ultrafiltration is evaluated for its feasibility in view of delivering adequate dialysis dose and fluid removal while minimizing the occurrence of adverse events. With this study, we follow the regulatory requirements of conducting post-market clinical follow-up to regularly and systematically collect clinical data on performance and safety over the entire life cycle of a medical device. ${ }^{17}$

\section{Materials and Methods Study Design and Objectives}

The objective of this study was to compare dialysis therapy performed with and without blood volume-controlled UF, the latter using two different HD systems. The clinical feasibility of combining body composition monitoring and blood volume-controlled UF was evaluated with respect to indicators of adequate dialysis, including dialysis dose (Kt/ V), ultrafiltration volume, fluid status and the occurrence of intradialytic morbid events.

The study used a combined retrospective and prospective, open-label, controlled, monocentric, and explorative design. It involved an intraindividual comparison of three treatment phases, each lasting four weeks, using retrospective clinical data from HD and on-line hemodiafiltration (HDF) treatments employing the 5008 therapy system with $\left(5008^{+}\right)$and without (5008-) the use of an integrated blood volume monitor (BVM). Upon enrollment, patients were switched to the 6008 CAREsystem, also using a BVM $(6008+)$. Both therapeutic systems are manufactured by Fresenius Medical Care, Bad Homburg, Germany. The major difference between the two systems is the design of the extracorporeal circuit, either as a conventional blood-line system (5008) or as an all-inone cassette system (6008), where the BVM cuvette is located in the arterial line or the arterial inflow to the cassette.

\section{Patients}

All patients were recruited at the Georg-Haas-Dialysis Center in Giessen, Germany. Adult patients who signed the informed consent form and were undergoing HD or HDF treatment with the 5008 therapy system three times a week with application of the BVM in UF control mode for at least four weeks before enrollment could be included. Furthermore, these patients must have been treated with the 5008 therapy system without the BVM for four weeks within two years before enrollment. 


\section{Blood Volume Monitoring and Treatments}

Blood volume monitoring (BVM) is an integrated function in both HD systems for monitoring relative blood volume (RBV) and managing the UF rate during treatment by employing feedback control. The BVM measures blood density based on the velocity of ultrasound pulses traveling through a cuvette with precise dimensions, which is part of the arterial blood line. ${ }^{18}$ As a consequence of ultrafiltration, blood density changes, and with that, ultrasound velocity, which makes it possible to derive blood volume relative to the pre-dialysis state. ${ }^{19,20}$

The UF control algorithm in the BVM implements the critical RBV concept, in which an automatic feedback loop controls the ultrafiltration rate (UFR) to continuously maintain the RBV above a predefined value for the respective treatment $\left(\mathrm{RBV}_{\text {crit }}\right)$. At treatment start, it applies twice the mean UFR as defined for the respective treatment. This rate is reduced as the session progresses, particularly when the RBV approaches the $\mathrm{RBV}_{\text {crit }}$ initially set by the user. ${ }^{16,20}$ If RBV rises above $R B V_{\text {crit }}$ due to vascular refilling, the UFR increases.

Patients received their regular treatment as prescribed by their attending physicians. Clinical practice at the study center was to set $\mathrm{RBV}_{\text {crit }}$ at $90 \%$ when a patient started treatment with blood volume-guided UF, and then to adjust upward or downward on an individual basis later, depending on whether the patient developed hypotonic episodes or was volume overloaded. Throughout the four-week study phase using the 6008 CAREsystem, $\mathrm{RBV}_{\text {crit }}$ and other treatment parameters should have been unchanged from the preceding treatment using the 5008 therapy system.

Retrospective data from each four-week treatment period with the 5008 therapy system, with and without applying the BVM, were recorded from available clinical records. During the four-week treatment using the 6008 CAREsystem with the BVM, data were collected prospectively.

The dialysis dose, $\mathrm{Kt} / \mathrm{V}$, was determined noninvasively for each treatment with On-line Clearance Monitoring $(\mathrm{OCM})^{21}$ integrated into both HD systems.

In addition, treatment data (blood flow rate, dialysate flow rate, treatment time, substitution flow rate in case of on-line HDF, mean ultrafiltration rate and ultrafiltration volume), pre- and post-dialysis blood pressure and heart rate were documented. Except for the HD system, no change in treatment parameters was foreseen when switching from $5008+$ to $6008+$.
Fluid status was assessed with bioimpedance spectroscopy using the Body Composition Monitor (BCM, Fresenius Medical Care, Bad Homburg, Germany). ${ }^{11,22}$ Routinely, the study center used to perform BCM measurements as clinically required to guide UF based on volume status. For the two retrospective phases of the study, single BCM measurements taken at any time during each of the four-week phases were extracted from the patient record. During the prospective phase, BCM measurements took place as single pre-dialysis measurements at the midweek session of week 4 .

Intradialytic morbid events (symptomatic hypotension (systolic blood pressure $<100 \mathrm{mmHg}$, associated with symptoms and interventions), muscle cramps, headache, nausea, dizziness, fatigue or other) and other adverse events were documented from each treatment.

\section{Ethical Conduct}

This study was started after approval by the competent ethics committee (Ethics Committee of Landesärztekammer Hessen/State Medical Association of Hesse, Approval No. FF103/218). All patients were informed orally and in writing of the purpose, conduct and risks of the study and were only enrolled after signing the informed consent form. The study was conducted in accordance with the principles of the Declaration of Helsinki.

\section{Trial Registration}

The study was registered with the Deutsches Register für Klinische Studien/German Clinical Trials Register (DRKS) with the following DRKS ID No.: DRKS00024720 on March 17, 2021.

\section{Statistical Analysis}

Analyses included all study patients for whom at least one documented treatment within the respective study phase with a valid outcome measurement was available. The safety analysis included all patients enrolled in the study.

Due to the exploratory nature of the study and its purpose as a post-market clinical follow-up study, no formal sample size estimation was performed. The target enrollment of 24 patients was mainly based on center feasibility.

A descriptive presentation of the parameters assessed during the study is provided by study phase. For continuous variables, mean and standard deviation are indicated; for categorical variables, absolute and relative frequencies 
are presented. Repeated measurements within the study phase were first averaged over all valid measurements by the patient and then descriptively summarized for the entire study population.

Study outcome parameters (Kt/V, UF volume, predialysis fluid overload) were compared exploratively employing a linear mixed model assuming no carryover effect. This model included a dichotomous treatment variable as a fixed effect for the comparisons of study phases $6008+$ and 5008+, as well as 5008- and 5008+. Random patient effects accounted for intercorrelations of repeated $\mathrm{Kt} / \mathrm{V}$ measurements in patients; one of them was nested within the fixed study phase to allow for larger intercorrelations of $\mathrm{Kt} / \mathrm{V}$ measurements within and across study phases.

No study hypothesis was formulated; therefore, all results, including $p$-values were interpreted on a purely exploratory basis.

Comparisons between study phases regarding safety parameters were based on descriptive statistics.

Statistical analysis was performed using SAS 9.4 (SAS Institute Inc., Cary, NC, USA).

\section{Results}

A total of 24 patients who previously underwent HD or on-line HDF with the 5008 therapy system and the BVM were enrolled in the study and switched to treatment with the 6008 CAREsystem. Data on HD treatments with the 5008 therapy system without the BVM were usable for 21 patients. Data from three patients were excluded because they were from treatments that did not take place within the previous two years or were not performed completely with the 5008 therapy system. The median interval between this study phase and enrollment in the prospective study phase was 5.8 months [range 2.7-18.3]. The patients were, on average, 76.5 years old, and $50 \%$ were male. The leading causes of chronic kidney disease were diabetes (33\%) and hypertension (17\%) (Table 1).

In all three treatment phases, the patients were predominantly treated with on-line HDF in post-dilution mode for more than four hours per treatment, with a mean blood flow rate between 344 and $360 \mathrm{~mL} / \mathrm{min}$ and an average substitution volume of 22 to 23 L (Table 2). During UF-controlled treatments (5008+, $6008+$ ), the average of the minimum RBV reached during the session was higher than the $\mathrm{RBV}_{\text {crit }}$ set by the user. This is also evident from the mean value of the individual treatment difference between $\mathrm{RBV}_{\text {crit }}$ and $\mathrm{RBV}_{\text {min }}$. Nevertheless, on average, $16.5 \%$ of treatments in the
Table I Patient Characteristics (at Time of Enrollment, Before Starting 6008+ Phase)

\begin{tabular}{|l|l|}
\hline Parameter & Value \\
\hline Age [years] & $76.5 \pm 9.1$ \\
\hline Sex [\% male] & $50 \%$ \\
\hline Dry body weight, male [kg] & $78.2 \pm 15.7$ \\
\hline Dry body weight, female [kg] & $74.7 \pm 14.0$ \\
\hline Body mass index [kg/m $\left.{ }^{2}\right]$ & $26.9 \pm 4.8$ \\
\hline Primary renal disease [n, \%]* & \\
Diabetes & $8,33 \%$ \\
Hypertension/large vessel disease & $4,17 \%$ \\
Glomerulonephritis & $2,8 \%$ \\
Interstitial nephritis/pyelonephritis & $1,4 \%$ \\
Transitional cell carcinoma & $1,4 \%$ \\
Glomerulonephropathy & $1,4 \%$ \\
Others/Unknown & $8,33 \%$ \\
\hline Comorbidities [n, \%]** & \\
Hypertension & $23,96 \%$ \\
Anemia & $23,96 \%$ \\
Secondary hyperparathyroidism & $20,83 \%$ \\
Hyperphosphatemia & $18,75 \%$ \\
Hyperlipidemia & $9,38 \%$ \\
Pain & $9,38 \%$ \\
Diabetes mellitus & $7,29 \%$ \\
Atrial fibrillation & $7,29 \%$ \\
Coronary artery disease & $7,29 \%$ \\
\hline Hypothyroidism & $6,24 \%$ \\
\hline
\end{tabular}

Notes: *More than one primary renal disease could be documented; $* *$ comorbidities are listed that were present in $\geq 25 \%$ of study patients.

Abbreviation: $\mathrm{n}$, number of patients.

5008+ phase and $7.9 \%$ of treatments in the 6008+ phase had an $\mathrm{RBV}_{\text {min }}$ that was below the $\mathrm{RBV}_{\text {crit }}$ specified for the respective treatment (Table 2).

The distribution of the adequacy parameter $\mathrm{Kt} / \mathrm{V}$ for the comparison of 5008-/5008+ and of 5008+/6008+ is shown in Figure 1. The comparison using a linear mixed model analysis yields similar results for treatments performed with the 5008 therapy system, with or without BVM (5008- vs $5008+, p=0.230)$. These intraindividual comparisons showed no significant differences with respect to the UF volume $(p=0.166)$ achieved and the predialysis fluid overload $(p=0.390)$, as assessed with the $\mathrm{BCM}$ during or at the end of the respective study phases (Table 3). The variability of post-dialysis body weight as a possible result of blood volume-controlled ultrafiltration did not differ between the study phases $(0.365 \mathrm{~kg}$ vs $0.428 \mathrm{~kg}$ for $5008-$ and $5008+$, respectively; $p=0.262$ ). 
Table 2 Treatment Parameters as Applied During the Three Treatment Phases (Mean \pm SD)

\begin{tabular}{|c|c|c|c|}
\hline & $5008-$ & $5008+$ & $6008+$ \\
\hline Parameter & $N=21$ & $N=24$ & $N=24$ \\
\hline On-line hemodiafiltration [\%] & 85.7 & 91.7 & 91.7 \\
\hline Effective treatment time [min] & $256 \pm 13$ & $250 \pm 15$ & $251 \pm 15$ \\
\hline Blood flow rate $[\mathrm{mL} / \mathrm{min}]$ & $344 \pm 45$ & $359 \pm 34$ & $360 \pm 37$ \\
\hline Dialysate flow rate $[\mathrm{mL} / \mathrm{min}]$ & $433 \pm 46$ & $449 \pm 49$ & $444 \pm 55$ \\
\hline Total treated blood volume $[\mathrm{L}]$ & $88.6 \pm 13.4$ & $90.5 \pm 11.0$ & $90.5 \pm 11.3$ \\
\hline Substitution flow rate* $[\mathrm{mL} / \mathrm{min}]$ & $95.6 \pm 10.7$ & $88.5 \pm 10.3$ & $92.0 \pm 13.8$ \\
\hline Total substitution volume* [L] & $23.2 \pm 5.8$ & $21.9 \pm 2.8$ & $22.8 \pm 3.7$ \\
\hline Prescribed ultrafiltration volume $[\mathrm{mL}]$ & $2211 \pm 800$ & $2287 \pm 789$ & $2480 \pm 810$ \\
\hline $\mathbf{R B V}_{\text {crit }}[\%]$ & $\mathrm{n} / \mathrm{a}$ & $87.4 \pm 2.1$ & $87.1 \pm 2.0$ \\
\hline $\mathbf{R B V}_{\min }[\%]$ & $\mathrm{n} / \mathrm{a}$ & $89.0 \pm 3.1$ & $89.1 \pm 2.5$ \\
\hline$\Delta \mathbf{R B V}_{\text {crit }}-\mathbf{R B V}_{\min }[\%]$ & $\mathrm{n} / \mathrm{a}$ & $-1.6 \pm 2.4$ & $-2.0 \pm 1.6$ \\
\hline \% sessions with $\mathbf{R B V}_{\min }<\mathbf{R B V}_{\text {crit }}$ & $\mathrm{n} / \mathrm{a}$ & $16.5 \pm 18.4$ & $7.9 \pm 9.6$ \\
\hline
\end{tabular}

Note: *From patients treated with on-line HDF.

Abbreviations: SD, standard deviation; n/a, not applicable.

With the 6008 CAREsystem, Kt/V comparable to the 5008 system, both employing blood volume-controlled UF, could be achieved ( $p=0.922$ ). The UF volume achieved was slightly and significantly higher with the 6008 CAREsystem
( $p=0.003$, Table 3 ), likely because of a higher prescribed UF volume (Table 2). Pre-dialysis fluid overload, as assessed with the BCM at the end of the study phases, did not differ between the two HD systems $(p=0.838)$.
A

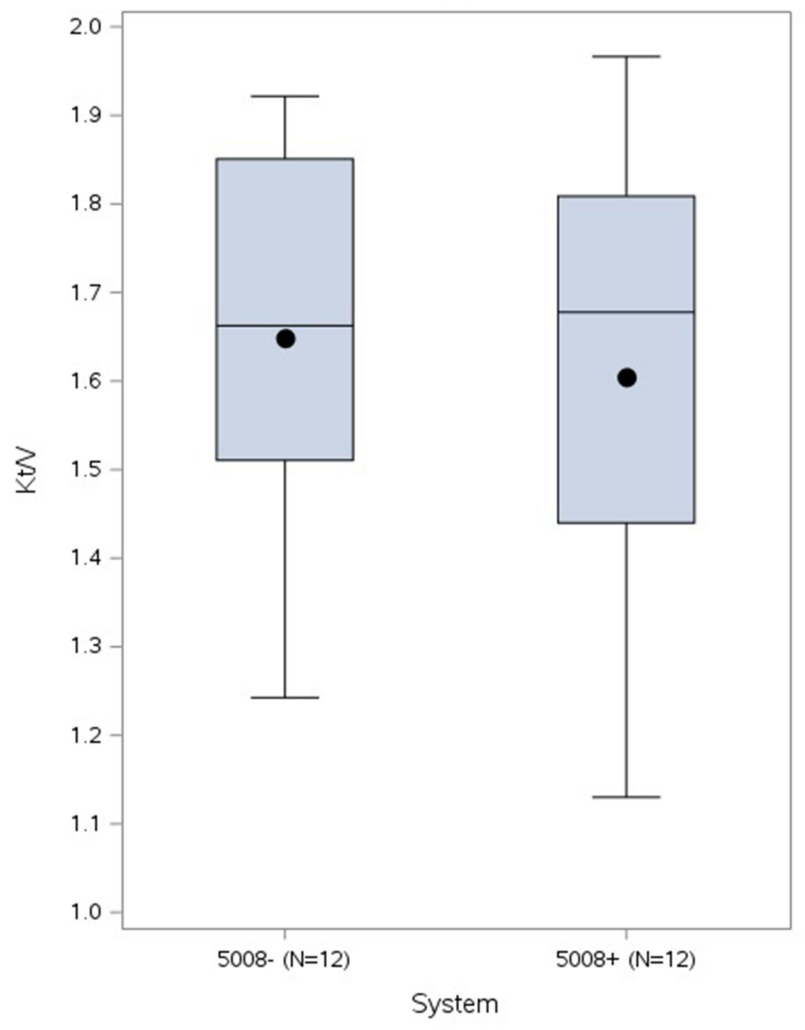

B

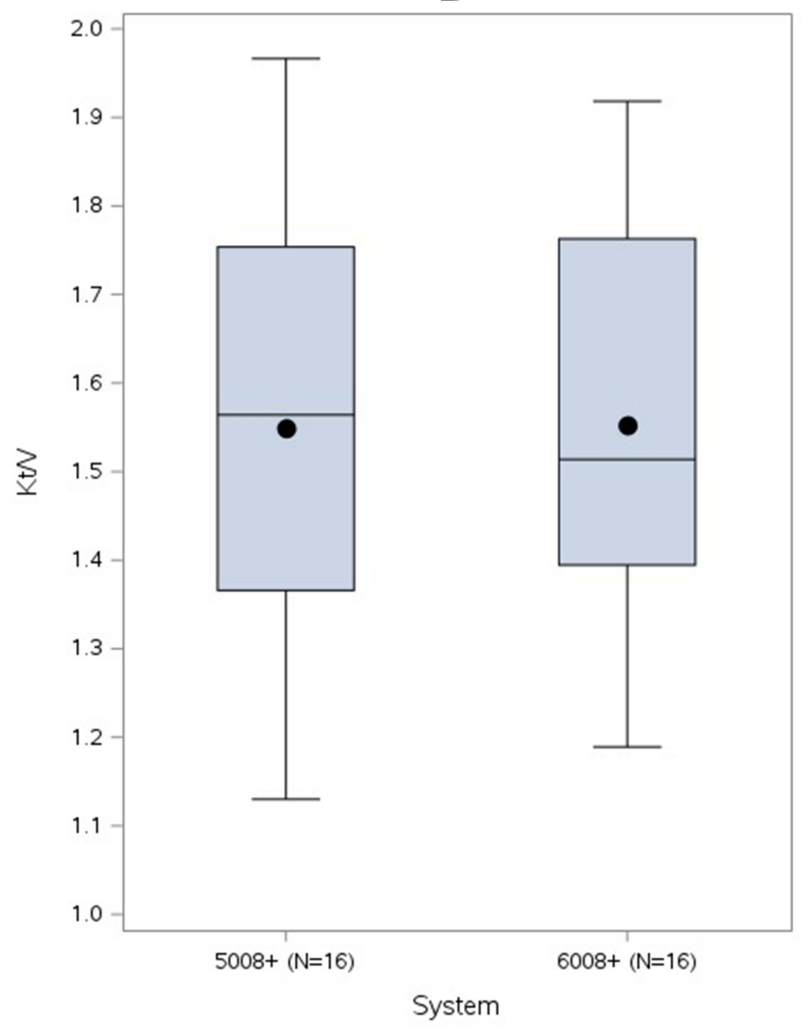

Figure I Dialysis dose (Kt/V) achieved with different treatment regimens. (A) Comparison of average Kt/V across treatments with and without use of the BVM (5008- vs $5008+)$; (B) comparison of average Kt/V across treatments with use of the BVM in different HD systems (5008+ vs 6008+). Displayed are the interquartile range (IQR; box), mean (dot), median (line), minimum and maximum values within IQR \pm I.5 IQR (whiskers). 
Table 3 Clinical Data on Treatment Efficacy and Fluid Status (Modeled Values, Estimate, SE)

\begin{tabular}{|c|c|c|c|c|c|c|}
\hline \multicolumn{7}{|c|}{ A: Comparison of Study Phases $5008-$ vs $5008+$} \\
\hline \multirow[b]{2}{*}{ Parameter } & \multirow[b]{2}{*}{$\mathrm{N}$} & \multicolumn{2}{|c|}{$5008-$} & \multicolumn{2}{|c|}{$5008+$} & \multirow{5}{*}{$\begin{array}{c}p \text {-value } \\
0.230 \\
0.166 \\
0.390\end{array}$} \\
\hline & & Estimate & SE & Estimate & SE & \\
\hline $\mathrm{Kt} / \mathrm{V}$ & 12 & 1.65 & 0.07 & 1.60 & 0.07 & \\
\hline UF volume achieved $[\mathrm{mL}]$ & 21 & 2189 & 169 & 2344 & 169 & \\
\hline Pre-dialysis fluid overload [mL] & 17 & 1912 & 324 & 2206 & 324 & \\
\hline \multicolumn{7}{|c|}{ B: Comparison of Study Phases $5008+$ vs $6008+$} \\
\hline & & \multicolumn{2}{|c|}{$5008+$} & \multicolumn{2}{|c|}{$6008+$} & \\
\hline Parameter & $\mathrm{N}$ & Estimate & SE & Estimate & SE & $p$-value \\
\hline $\mathrm{Kt} / \mathrm{V}$ & 16 & 1.55 & 0.06 & 1.55 & 0.06 & 0.922 \\
\hline UF volume achieved [mL] & 24 & 2316 & 154 & 2492 & 154 & 0.003 \\
\hline Pre-dialysis fluid overload [mL] & 23 & 2035 & 277 & 1987 & 277 & 0.838 \\
\hline
\end{tabular}

Abbreviation: SE, standard error.

Mean systolic blood pressure decreased from start to end of the treatment in all three study phases by an average of $-3.2,-10.9$ and $-5.3 \mathrm{mmHg}$ during the $5008^{-}, 5008+$ and $6008+$ treatment phases, respectively. The decrease in diastolic blood pressure, heart rate and body weight throughout the treatment was similar in all three study phases (Table 4).

From the three study phases, 97 adverse events in total were documented (5008-: 24, 5008+: 30,6008+: 43). Out of them, only 6,9 , and 11 events occurred during the treatments, but all were assessed by the investigator as unrelated to the medical device or treatment. Intradialytic morbid events occurred overall at low frequency, in less than $3 \%$ of treatments in all study phases. Intradialytic hypotension occurred in two and three treatments during the study phases using the 5008 therapy system, with and without the BVM, respectively, and in no treatment during the study phase using the 6008 CAREsystem (Table 5).

\section{Discussion}

This study achieved its objective of showing that clinical practice employing bioimpedance spectroscopy and hemodialysis and hemodiafiltration with blood volumecontrolled ultrafiltration (UF) provides adequate dialysis dose and fluid removal while maintaining acceptable fluid status and low frequency of intradialytic morbid events. The

Table 4 Clinical Parameters by Study Phase

\begin{tabular}{|c|c|c|c|c|}
\hline & & 5008- & $5008+$ & $6008+$ \\
\hline \multicolumn{2}{|l|}{ Number of patients } & 21 & 24 & 24 \\
\hline \multirow[t]{3}{*}{ Systolic blood pressure $[\mathrm{mmHg}]$} & Pre-dialysis & $143 \pm 18$ & $142 \pm 19$ & $140 \pm 19$ \\
\hline & Post-dialysis & $|40 \pm 2|$ & $131 \pm 19$ & $134 \pm 15$ \\
\hline & Change & $-3.2 \pm 14$ & $-10.9 \pm 15$ & $-5.3 \pm 15$ \\
\hline \multirow[t]{3}{*}{ Diastolic blood pressure $[\mathrm{mmHg}]$} & Pre-dialysis & $60 \pm 11$ & $59 \pm 12$ & $68 \pm 10$ \\
\hline & Post-dialysis & $62 \pm 11$ & $61 \pm 11$ & $67 \pm 10$ \\
\hline & Change & $2 \pm 6$ & $2 \pm 6$ & $-0.6 \pm 6$ \\
\hline \multirow[t]{3}{*}{ Heart rate $[\mathrm{I} / \mathrm{min}]$} & Pre-dialysis & $70 \pm 11$ & $66 \pm 8$ & $67 \pm 9$ \\
\hline & Post-dialysis & $71 \pm 12$ & $68 \pm 8$ & $69 \pm 9$ \\
\hline & Change & $1 \pm 9$ & $2 \pm 6$ & $2 \pm 6$ \\
\hline \multirow[t]{3}{*}{ Body weight $[\mathrm{kg}]$} & Pre-dialysis & $80 \pm 15$ & $79 \pm 15$ & $79 \pm 16$ \\
\hline & Post-dialysis & $78 \pm 15$ & $77 \pm 15$ & $77 \pm 15$ \\
\hline & Change & $-1.7 \pm 0.8$ & $-1.9 \pm 0.7$ & $-2.0 \pm 0.7$ \\
\hline
\end{tabular}


Table 5 Frequency of Intradialytic Morbid Events (IMEs)

\begin{tabular}{|l|c|c|c|}
\hline & $\mathbf{5 0 0 8 -}$ & $\mathbf{5 0 0 8 +}$ & $\mathbf{6 0 0 8 +}$ \\
\hline Number of valid treatments & 247 & 281 & 281 \\
\hline Type of IME & & & \\
Symptomatic hypotension & 2 & 3 & - \\
Muscle cramps & 1 & 2 & 3 \\
Dizziness & - & - & 1 \\
Vomiting & - & - & 1 \\
Anticoagulation-related problems & 2 & 1 & 2 \\
Other & 1 & 2 & - \\
\hline Total (N, \% of treatments) & $\mathbf{6 ~ ( 2 . 4 \% ) ~}$ & $\mathbf{8 ~ ( 2 . 8 \% ) ~}$ & $\mathbf{7 ~ ( 2 . 5 \% ) ~}$ \\
\hline
\end{tabular}

dialysis center followed a strategy to define and achieve dry weight and UF goals through body composition monitoring in combination with blood volume-controlled UF. The target UF volume, the UFR profile and plasma refilling rates are associated with variations in the slope of blood volume decrease along the duration of the treatment and show further intraindividual and interindividual dependency on individual patient and treatment-related factors. Whereas plasma refilling is expected to be more pronounced at the start of treatment when a patient is volume overloaded, it might decelerate along with fluid removal and with approaching dry weight toward the end of treatment. Therefore, the individual patient trajectory of blood volume may be influenced, among other factors, by hydration status at the start of dialysis. Volume-expanded patients tend toward a flat line of blood volume, whereas with less volume overload, the slope of blood volume decrease during treatment becomes steeper, ${ }^{19,23,24}$ even if the association was not confirmed in another study. ${ }^{25}$ Another study differentiated the change in relative blood volume (RBV) as associated with the pre-dialysis volume status in patients with moderate volume overloads and UF rates, while vascular refilling was rather controlled by the UF rate. ${ }^{26}$

The algorithm in the blood volume monitoring (BVM) device starts, by definition, with an UFR twice the mean UFR as defined at the start of the respective treatment. ${ }^{16}$ This provides space to downregulate the UFR responding to an $\mathrm{RBV}$ approaching the $\mathrm{RBV}_{\text {crit }}$, without compromising the overall goal of fluid removal. In this way, fluid removal could be achieved within the planned treatment time of about four hours. This avoids UF rates that cause excessive intravascular volume depletion, which puts hemodynamic stability at risk ${ }^{26}$ and which are associated with accelerated decline in residual kidney function and elevated mortality risk. ${ }^{9,27}$ Although our study only covered four-week observation phases, this therapeutic approach makes it possible to identify whether changes in body weight over longer periods are caused by changes in volume status, in order to adapt treatment targets individually.

The patients reached, on average, a minimum RBV of $89 \%$. This value falls in the range of RBV that is associated with a hazard ratio $<1$ for mortality in an analysis of RBV and its association with survival. ${ }^{28}$ Although our study was not designed to assess long-term effects, both the treatment settings to keep RBV within a favorable range and the resulting low frequency of intradialytic hypotension may contribute to a good long-term prognosis in this patient cohort.

The Kt/V level delivered was comparable between the three treatment approaches and was, in each phase, higher than the recommended minimum level of single pool Kt/V of $1.4,{ }^{29}$ even though the On-line Clearance Monitoring (OCM) measurement of $\mathrm{Kt} / \mathrm{V}$ tends to underestimate the real value. ${ }^{30}$ Delivery of the dialysis dose was thus unaffected by the use of blood volume-guided UF control, as also described earlier, ${ }^{31}$ and with overall low incidence in our study, not affected by intradialytic hypotension, as previously described. ${ }^{32}$ This is not entirely unexpected because factors determining the dialysis dose achieved, such as blood flow and treatment time in view of clearance, as well as the patient's urea distribution volume and volume change after dialysis, ${ }^{33}$ have been comparable between the treatment settings investigated.

The clinical usefulness of UF control using the BVM to support hemodynamic stability has previously been studied, and it has been shown that the frequency of treatments affected by intradialytic morbid events is reduced in hypotension-prone patients. ${ }^{31,34}$ Proneness to intradialytic hypotension was not an inclusion criterion, which explains why we observed fewer intradialytic morbid events overall and less decrease in systolic blood pressure from pre- to post-dialysis than in previous studies. In the present study cohort, informed decisions on fluid management based on body composition measurements are possible, which was not the case in previous studies.

It must be acknowledged that not only UF but also other treatment-related factors, such as dialysate sodium ${ }^{35}$ and dialysate temperature, ${ }^{36}$ relate to intradialytic hemodynamic stability; however, these factors are intertwined, and change in blood volume plays a central role in the physiological pathway on which all these factors contribute to the development of intradialytic hypotension. ${ }^{5}$ 
In our study, the frequency of treatments involving intradialytic morbid events (IMEs), particularly intradialytic hypotension, was very low. Whereas the frequency of IMEs is often between $5 \%$ and $30 \%{ }^{37}$ and may even exceed this range, depending on the definition of intradialytic hypotension, ${ }^{6}$ we see less than $3 \%$ of treatments being affected by intradialytic morbid events, regardless of whether UF control was used. The frequency of hypotensive episodes was $0.6 \%$, far lower than the $19-21 \%$ in the patient cohorts of the HEMO study and from a large dialysis provider applying corresponding definitions of hypotensive episodes. ${ }^{6}$ Our observation of good hemodynamic stability achieved with the approach studied can, moreover, be considered a prerequisite for safely performing long nocturnal and home treatments, which we include in our treatment offerings to satisfy patient preferences.

Blood volume-controlled ultrafiltration was seen as inconclusive with respect to long-term patient outcomes by the European Best Practice Guidelines (EBPG) working group on hemodynamic stability. However, based on the demonstrated benefit regarding intradialytic hypotension (IDH), the use of automatic blood volume control is suggested as a second-line option to prevent IDH in patients with refractory IDH. ${ }^{38}$ Therefore, blood volumecontrolled UF is not considered as an isolated measure but as one that contributes together with other physiological treatment options, including treatment modality, dialysate temperature and dialysate composition, to prevent IDH and reduce the associated mortality risk. ${ }^{38}$

This study certainly has limitations. Using both retrospective and prospective data may not exclude data collection under different conditions, although this risk should be manageable because the center operates using stable processes with a stable clinical staff. This was a monocentric study, performed in a limited-care setting, which might exclude some markedly unstable patients and could explain, at least in part, the low complication rate during treatments. The predominantly performed on-line HDF modality may as well contribute to hemodynamic stability with overall low frequency of intradialytic hypotension. ${ }^{39}$ Based on this experience, further studies should be conducted to demonstrate the clinical benefit of blood volume-controlled UF and of monitoring volume status, particularly in patients with difficulties maintaining euvolemia and those with cardiovascular comorbidities, as well as hypotension-prone patients.

\section{Conclusion}

This study illustrates a clinical approach combining regular tracking of volume status and management of intradialytic fluid removal through blood volume-controlled ultrafiltration. Together with additional means to provide optimal extracorporeal kidney replacement therapy, it allows to deliver an adequate dialysis dose and fluid removal to maintain volume status and low incidence of intradialytic morbid events. Such physiological feedback regulation enables long-term and personalized management of kidney replacement therapy.

\section{Abbreviations}

BCM, Body Composition Monitor; BVM, Blood Volume Monitor; EBPG, European Best Practice Guidelines; HD, Hemodialysis; HDF, Hemodiafiltration; HEMO Study, Hemodialysis study; IDH, Intradialytic hypotension; IME, Intradialytic morbid event; KRT, Kidney replacement therapy; Kt/V, Dialysis dose; OCM, On-line Clearance Monitoring; RBV, Relative blood volume; $\mathrm{RBV}_{\text {crit }}$ Critical relative blood volume; UF, Ultrafiltration; UFR, Ultrafiltration rate.

\section{Data Sharing Statement}

The datasets used and/or analyzed during the current study are available from the corresponding authors on reasonable requests.

\section{Author Contributions}

All authors made substantial contributions to conception and design, acquisition of data, or analysis and interpretation of data and took part in drafting the article or revising it critically. All authors agreed to submit the article to the current journal, gave final approval for the version to be published and agreed to be accountable for all aspects of the work.

\section{Funding}

The study was funded by Fresenius Medical Care Deutschland GmbH.

\section{Disclosure}

MSG, AG and JB are full-time employees of Fresenius Medical Care. The authors report no other conflicts of interest in this work. 


\section{References}

1. Hecking M, Moissl U, Genser B, et al. Greater fluid overload and lower interdialytic weight gain are independently associated with mortality in a large international hemodialysis population. Nephrol Dial Transplant. 2018;33(10):1832-1842. doi:10.1093/ndt/gfy083

2. Zoccali C, Moissl U, Chazot C, et al. Chronic fluid overload and mortality in ESRD. J Am Soc Nephrol. 2017;28(8):2491-2497. doi:10.1681/ASN.2016121341

3. Flythe JE, Bansal N. The relationship of volume overload and its control to hypertension in hemodialysis patients. Semin Dial. 2019;32 (6):500-506. doi:10.1111/sdi.12838

4. Loutradis C, Sarafidis PA, Ekart R, et al. The effect of dry-weight reduction guided by lung ultrasound on ambulatory blood pressure in hemodialysis patients: a randomized controlled trial. Kidney Int. 2019;95(6):1505-1513. doi:10.1016/j.kint.2019.02.018

5. Sars B, van der Sande FM, Kooman JP. Intradialytic hypotension: mechanisms and outcome. Blood Purif. 2020;49(1-2):158-167. doi: $10.1159 / 000503776$

6. Flythe JE, Xue H, Lynch KE, Curhan GC, Brunelli SM. Association of mortality risk with various definitions of intradialytic hypotension. $J \mathrm{Am}$ Soc Nephrol. 2015;26(3):724-734. doi:10.1681/ASN.2014020222

7. Chou JA, Streja E, Nguyen DV, et al. Intradialytic hypotension, blood pressure changes and mortality risk in incident hemodialysis patients. Nephrol Dial Transplant. 2018;33(1):149-159. doi:10.1093/ndt/gfx037

8. Assimon MM, Wenger JB, Wang L, Flythe JE. Ultrafiltration rate and mortality in maintenance hemodialysis patients. Am J Kidney Dis. 2016;68(6):911-922. doi:10.1053/j.ajkd.2016.06.020

9. Saran R, Bragg-Gresham JL, Levin NW, et al. Longer treatment time and slower ultrafiltration in hemodialysis: associations with reduced mortality in the DOPPS. Kidney Int. 2006;69(7):1222-1228. doi:10.1038/sj.ki.5000186

10. van der Sande FM, van de Wal-visscher ER, Stuard S, Moissl U, Kooman JP. Using bioimpedance spectroscopy to assess volume status in dialysis patients. Blood Purif. 2020;49(1-2):178-184. doi: $10.1159 / 000504079$

11. Chamney PW, Wabel P, Moissl UM, et al. A whole-body model to distinguish excess fluid from the hydration of major body tissues. $\mathrm{Am}$ J Clin Nutr. 2007;85(1):80-89. doi:10.1093/ajen/85.1.80

12. Zoccali C. Lung Ultrasound in the Management of Fluid Volume in Dialysis Patients: potential Usefulness. Semin Dial. 2017;30(1):6-9. doi:10.1111/sdi.12559

13. Stenberg J, Melin J, Lindberg M, Furuland H. Brain natriuretic peptide reflects individual variation in hydration status in hemodialysis patients. Hemodial Int. 2019;23(3):402-413. doi:10.1111/hdi.12751

14. Wizemann V, Schilling M. Dilemma of assessing volume state-the use and the limitations of a clinical score. Nephrol Dial Transplant. 1995; 10(11):2114-2117.

15. Kron S, Schneditz D, Leimbach T, Czerny J, Aign S, Kron J. Determination of the critical absolute blood volume for intradialytic morbid events. Hemodial Int. 2016;20(2):321-326. doi:10.1111/ hdi. 12375

16. Kraemer M. New strategies for reducing intradialytic symptoms. Semin Dial. 1999;12(5):389-395. doi:10.1046/j.1525-139X.1999.99058.x

17. Regulation (EU) $2017 / 745$ on Medical Devices. European parliament and the council of the European Union, 5 April 2017; 2017.

18. Johner C, Chamney PW, Schneditz D, Kramer M. Evaluation of an ultrasonic blood volume monitor. Nephrol Dial Transplant. 1998;13 (8):2098-2103. doi:10.1093/ndt/13.8.2098

19. Dasselaar JJ, van der Sande FM, Franssen CF. Critical evaluation of blood volume measurements during hemodialysis. Blood Purif. 2012;33(1-3):177-182. doi:10.1159/000334142

20. Schneditz D, Pogglitsch H, Horina J, Binswanger U. A blood protein monitor for the continuous measurement of blood volume changes during hemodialysis. Kidney Int. 1990;38(2):342-346. doi:10.1038/ ki.1990.207
21. Goldau R, Kuhlmann U, Samadi N, et al. Ionic dialysance measurement is urea distribution volume dependent: a new approach to better results. Artif Organs. 2002;26(4):321-332. doi:10.1046/j.1525-1594.2002.06817.x

22. Moissl UM, Wabel P, Chamney PW, et al. Body fluid volume determination via body composition spectroscopy in health and disease. Physiol Meas. 2006;27(9):921-933. doi:10.1088/0967$3334 / 27 / 9 / 012$

23. Steuer RR, Germain MJ, Leypoldt JK, Cheung AK. Enhanced fluid removal guided by blood volume monitoring during chronic hemodialysis. Artif Organs. 1998;22(8):627-632. doi:10.1046/ j.1525-1594.1998.06036.x

24. Maduell F, Arias M, Masso E, et al. Sensitivity of blood volume monitoring for fluid status assessment in hemodialysis patients. Blood Purif. 2013;35(1-3):202-208. doi:10.1159/000346630

25. Keane DF, Baxter P, Lindley E, Rhodes L, Pavitt S. Time to reconsider the role of relative blood volume monitoring for fluid management in hemodialysis. ASAIO J. 2018;64(6):812-818. doi:10.1097/ MAT.0000000000000795

26. Kron S, Schneditz D, Leimbach T, Aign S, Kron J. Vascular refilling is independent of volume overload in hemodialysis with moderate ultrafiltration requirements. Hemodial Int. 2016;20(3):484-491. doi:10.1111/hdi.12417

27. Lee YJ, Okuda Y, Sy J, et al. Ultrafiltration rate, residual kidney function, and survival among patients treated with reduced-frequency hemodialysis. Am J Kidney Dis. 2020;75(3):342-350. doi:10.1053/j. ajkd.2019.08.019

28. Preciado P, Zhang H, Thijssen S, Kooman JP, van der Sande FM, Kotanko P. All-cause mortality in relation to changes in relative blood volume during hemodialysis. Nephrol Dial Transplant. 2019;34(8):1401-1408. doi:10.1093/ndt/gfy286

29. National Kidney Foundation. KDOQI clinical practice guideline for hemodialysis adequacy: 2015 update. Am J Kidney Dis. 2015;66 (5):884-930.

30. Ahrenholz P, Taborsky P, Bohling M, et al. Determination of dialysis dose: a clinical comparison of methods. Blood Purif. 2011;32 (4):271-277. doi:10.1159/000330340

31. Gabrielli D, Krystal B, Katzarski K, et al. Improved intradialytic stability during haemodialysis with blood volume-controlled ultrafiltration. J Nephrol. 2009;22(2):232-240.

32. Ronco C, Brendolan A, Milan M, Rodeghiero MP, Zanella M, La Greca G. Impact of biofeedback-induced cardiovascular stability on hemodialysis tolerance and efficiency. Kidney Int. 2000;58 (2):800-808. doi:10.1046/j.1523-1755.2000.00229.x

33. Brunelli S. The dialysis prescription. In: Nissenson AR, Fine RN, editors. Handbook of Dialysis Therapy. Philadelphia, PA: Elsevier; 2017.

34. Garzoni D, Keusch G, Kleinoeder T, et al. Reduced complications during hemodialysis by automatic blood volume controlled ultrafiltration. Int J Artif Organs. 2007;30(1):16-24. doi:10.1177/ 039139880703000104

35. de Paula FM, Peixoto AJ, Pinto LV, Dorigo D, Patricio PJ, Santos SF. Clinical consequences of an individualized dialysate sodium prescription in hemodialysis patients. Kidney Int. 2004;66(3):1232-1238. doi:10.1111/j.1523-1755.2004.00876.x

36. van der Sande FM, Kooman JP, van Kuijk WH, Leunissen KM. Management of hypotension in dialysis patients: role of dialysate temperature control. Saudi J Kidney Dis Transpl. 2001;12 (3):382-386

37. Chou JA, Kalantar-Zadeh K, Mathew AT. A brief review of intradialytic hypotension with a focus on survival. Semin Dial. 2017;30 (6):473-480. doi:10.1111/sdi.12627

38. Kooman J, Basci A, Pizzarelli F, et al. EBPG guideline on haemodynamic instability. Nephrol Dial Transplant. 2007;22(Suppl 2):ii22ii44. doi:10.1093/ndt/gfm019

39. Patel S, Raimann JG, Kotanko P. The impact of dialysis modality and membrane characteristics on intradialytic hypotension. Semin Dial. 2017;30(6):518-531. doi:10.1111/sdi.12636 


\section{Publish your work in this journal}

The International Journal of Nephrology and Renovascular Disease is an international, peer-reviewed open-access journal focusing on the pathophysiology of the kidney and vascular supply. Epidemiology, screening, diagnosis, and treatment interventions are covered as well as basic

Submit your manuscript here: https://www.dovepress.com/international-journal-of-nephrology-and-renovascular-disease-journal science, biochemical and immunological studies. The manuscript management system is completely online and includes a very quick and fair peer-review system, which is all easy to use. Visit http://www.dovepress.com/testimonials.php to read real quotes from published authors. 